\title{
Separação de levedura da indústria de cerveja utilizando mini-hidrociclone
}

Beer industry yeast separation using mini-hydrocyclone

\author{
1 Carla Torres Dias \\ 1 Vitor Araújo de Souza Franco \\ 1 Luiz Gustavo Dias Camargos \\ 1 Marcelo da Silva Batista marcelobatista@ufsj.edu.br \\ 2 Roberta Viana Ferreira
}

1 Universidade Federal de São João del-Rei, Ouro Branco, MG.

2 Centro Federal de Educação Tecnológica de Minas Gerais, Belo Horizonte, MG.

\begin{abstract}
Resumo
A separação da levedura do mosto da indústria de cerveja utilizando hidrociclones é uma alternativa para redução de custos com o uso de centrífugas. Este trabalho teve como objetivo avaliar a eficiência de separação de leveduras do mosto de indústrias cervejeiras utilizando mini-hidrociclones da família Mozley1. Os hidrociclones HC090, HC100, HC110 e HC120 foram construídos em plástico ABS, com Dc entre 9 e $12 \mathrm{~mm}$, usando impressora 3D. A eficiência de separação dos mini-hidrociclones foi avaliada em relação ao teor de sólido obtido na saída do underflow e overflow. Os resultados mostraram que a impressão 3D é uma técnica rápida e barata para se construir equipamentos para experimentos usando engenharia. A eficiência total de separação variou amplamente para os hidrociclones HC090 (50-93\%), HC100 (35-95\%), HC110 (43-79\%) e HC120 (33-96\%). A eficiência total reduzida foi proporcional em relação Du/Dc de construção dos hidrociclones: $\mathrm{HCO} 90>\mathrm{HC} 100>\mathrm{HC} 110>\mathrm{HC} 120$. Os hidrociclones mostraram-se promissores para a separação de leveduras do mosto da indústria cervejeira.
\end{abstract}

\section{Palavras-chave}

Hidrociclone. Levedura. Separação. Indústria de cerveja.

\begin{abstract}
Yeast separation of the fermented from beer industry using hydrocyclones is an alternative for cost reduction when using centrifuges. This study aimed to evaluate the yeast separation efficiency of the fermented industries using mini-hydrocyclones of Mozley1 family. The hydrocyclones HCO90, HC100, HC1 10 and HC1 20 were constructed from ABS plastic, Dc between 9 and $12 \mathrm{~mm}$, using $3 D$ printer. Separation efficiency of the mini-hydrocyclones was evaluated by relation to the solid content obtained in the underflow and overflow. The results showed that $3 D$ printing is a fast and inexpensive technique to build equipment for experiments using engineering. Total separation efficiency varied widely for hydrocyclones HCO90 (5093\%), HC100 (35-95\%), HC110 (43-79\%) and HC120 (33-96\%). Reduced efficiency was proportional to the relationship Du/Dc of the hydrocyclones construction: HCO9O> HC100> HC110> HC120. The hydrocyclones proved promising for yeast separation from fermented.
\end{abstract}

\section{Keywords}

Hydrocyclone. Yeast. Separation. Beer industry.

\section{Como você deve citar?}

BATISTA, Marcelo da Silva et al. Separação de levedura da indústria de cerveja utilizando mini-hidrociclone. Cadernos UniFOA, Volta Redonda, n. 36, p. 37-45, abr. 2018. 


\section{INTRODUÇÃO}

A indústria cervejeira brasileira produz 14,1 bilhões de litros de cerveja por ano e contribui com $1,6 \%$ do PIB nacional. No processo de produção de cerveja, utiliza-se água tratada, malte, lúpulo, levedura e cereais como: milho, arroz e trigo, que também podem ser usados para substituir parcialmente o malte (CERVBRASIL, 2016). Nesse processo, a água rica em açúcares, denominada mosto, passa pela etapa de fermentação (5 a 10 dias) usando leveduras. Após a fermentação, faz-se necessário a separação da levedura do mosto para reaproveitamento em outra batelada de fermentação. Na maioria das indústrias, a separação da levedura do mosto fermentado é realizada com o uso de centrífugas que apresentam características como: dispendiosidade, alto consumo de energia e necessidade constante de manutenção (BICALHO, 2011). Devido a esses fatores, os hidrociclones apresentam-se como alternativa para separação de levedura, pois se destacam por serem compactos, não possuírem partes móveis, requererem baixa manutenção mecânica e baixo consumo de energia e, ainda, apresentarem baixos custos de confecção e instalação (ARAÚJO et al., 2015). O princípio de funcionamento dos hidrociclones deve-se à alimentação tangencial da suspensão sólido-líquido na região cilíndrica do equipamento, 0 que provoca a formação de vórtex, fazendo com que as partículas maiores e mais densas sejam projetadas contra a parede, sendo então arrastadas até a saída inferior (underflow). Em contrapartida, as partículas menores e de menor densidade são projetadas para o centro do equipamento passando pelo vórtex em sentido ascendente e saindo pelo orifício superior (overflow) (YU et al., 2017; NI et al., 2017).

A eficiência de separação de partículas em um hidrociclone depende da geometria do equipamento, das propriedades físicas do sólido, do fluido e das condições de operação. Diversos estudos sobre o efeito das proporções geométricas do equipamento na eficiência de separação em hidrociclones são encontrados na literatura (BICALHO, 2011; BICALHO et al., 2012; ARAÚJO et al., 2015; NI et al., 2018). Geralmente, essas proporções geométricas são caracterizadas pelas dimensões desses equipamentos em relação ao diâmetro da parte cilíndrica (Dc), que é definido em função do diâmetro das partículas que se pretende separar (SYED et al., 2017). Como o diâmetro médio das células de Saccharomyces cerevisiae é de $9 \mu \mathrm{m}$, o Dc ideal de hidrociclones para separação dessas leveduras deve estar entre 6,3 e 12,7 mm (EVANS et al., 2015; SYED et al., 2017).

O presente trabalho teve como objetivo avaliar a eficiência de separação de leveduras (Saccharomyces cerevisiae) do mosto de indústrias cervejeiras, utilizando mini-hidrociclones com Dc entre 9 e 12 mm (construídos em impressora 3D). A eficiência de separação dos mini-hidrociclones foi avaliada em relação ao teor de sólido obtido na saída do underflow e overflow.

\section{METODOLOGIA}

\subsection{Caracterização do mosto de cerveja}

O mosto em fermentação foi cedido pela Cervejaria Walls e pela Cervejaria Loba, localizadas, respectivamente, nas cidades de Belo Horizonte - MG e Santana dos Montes - MG. O material recebido foi misturado, homogeneizado e estocado a baixas temperaturas $\left(<4^{\circ} \mathrm{C}\right)$. A caracterização do mosto de cerveja foi realizada por medidas de $\mathrm{pH}$, densidade e teor de sólidos totais. $\mathrm{O}$ valor de $\mathrm{pH}$ foi medido com fitas indicadoras e a densidade aferida por picnometria na temperatura de $24^{\circ} \mathrm{C}$. 0 teor de sólidos no mosto foi calculado pela diferença de massa úmida e seca. Para obter a massa seca, o mosto foi secado em estufa a $90^{\circ} \mathrm{C}$ por $2 \mathrm{~h}$, utilizando-se placa de Petri. 


\subsection{Construção dos mini-hidrociclones}

Os mini-hidrociclones da família Mozley 1 foram projetados em um software em ambiente 3D (Figura 1). Posteriormente, os mini-hidrociclones foram construídos em uma impressora 3D, modelo XYZ printing 1.0, utilizando-se como matéria-prima o plástico ABS. Devido às limitações operacionais (que necessitava que o hidrociclone estivesse apoiado sobre a plataforma da impressora), as impressões foram realizadas com a parte superior do hidrociclone voltada para baixo. Dessa maneira, os dutos de alimentação e do overflow foram apoiados e a estrutura permaneceu estável durante todo o processo de impressão. Os apoios desses dutos foram removidos, após o processo de impressão, para fixação de mangueiras de silicone nas devidas conexões (alimentação e overflow).

Figura 1 - Representação dos mini-hidrociclones: (a) Corte transversal na região cilíndrica, (b) corte longitudinal, (c) hidrociclone completo, (d) características gerais.

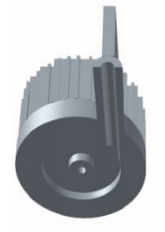

(a)
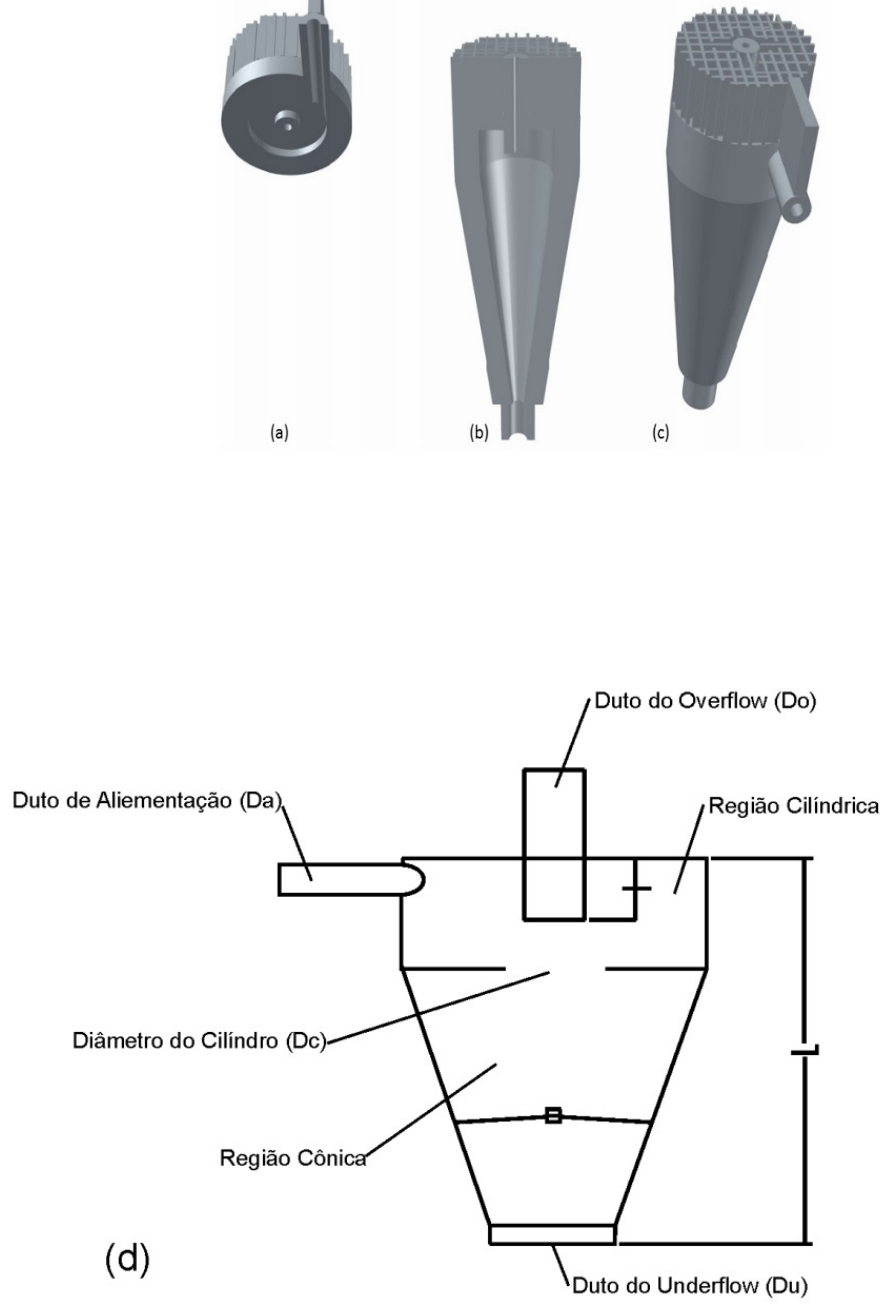

Fonte: dos autores, 2018.

Os mini-hidrociclones foram construídos conforme as dimensões apresentadas na Tabela 1. Foram construídos quatro hidrociclones com diâmetros da parte cilíndrica (Dc) igual a $9 \mathrm{~mm}$ (HC090), 10 mm (HC100), 11 mm (HC110) e 12 mm (HC120). 
Tabela 1 - Dimensões dos mini-hidrociclones.

\begin{tabular}{lllll}
\hline Parâmetro & HCO90 & HC100 & HC110 & HC120 \\
\hline Dc (mm) & 9,0 & 10,0 & 11,0 & 12,0 \\
Da (mm) & 1,4 & 1,5 & 1,7 & 1,8 \\
Do $(\mathrm{mm})$ & 1,9 & 2,1 & 2,4 & 2,6 \\
L (mm) & 66,9 & 74,3 & 81,7 & 89,2 \\
I (mm) & 5,1 & 5,7 & 6,3 & 6,8 \\
h* (mm) & 61,7 & 68,6 & 75,5 & 82,3 \\
$\Theta$ & 6,0 & 6,0 & 6,0 & 6,0 \\
\hline
\end{tabular}

*h $=\mathrm{L}-\mathrm{I}$, distância entre o tubo de underflow (apex) e a entrada do overflow (vortex).

Fonte: dos autores, 2018.

\subsection{Processo de separação}

Na Figura 2 pode ser observada a representação da unidade experimental construída no Laboratório de Engenharia Química da Universidade Federal de São João del Rei. Nessa figura são mostrados os equipamentos e materiais (tubos de PVC de $34^{\prime}$, mangueiras de silicone, etc.) utilizados na montagem da unidade.

Figura 2 - Representação da unidade experimental

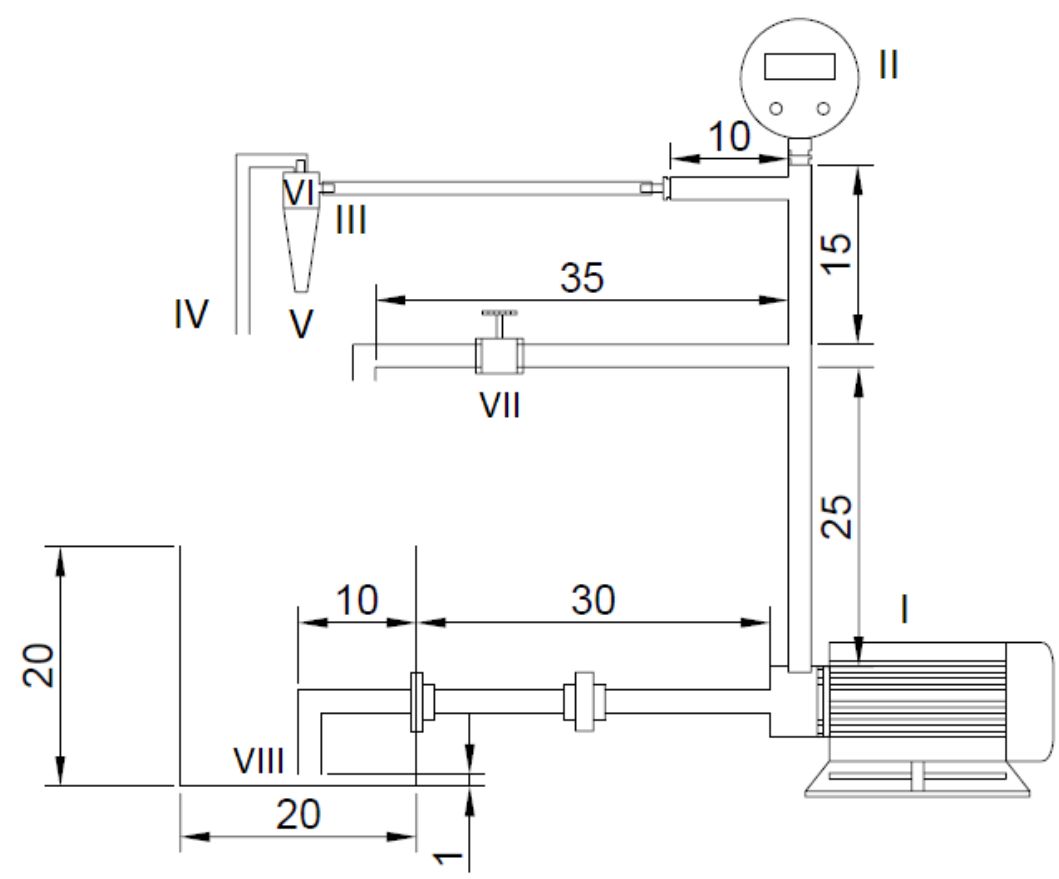

I. Bomba Centrífuga (0,5 Cv); II. Manômetro digital (0 a 199 psi); III. Alimentação do hidrociclone; IV. Tubulação de Overflow; V. Tubulação de Underflow; VI. Hidrociclone; VII. Válvula de controle de reciclo; VIII. Reservatório de cerveja.

Fonte: dos autores, 2018

A tubulação oriunda do recalque da bomba é dividida em duas correntes: uma linha de reciclo e outra linha de alimentação do hidrociclone. A vazão de entrada no hidrociclone foi controlada através de uma válvula e a homogeneização do mosto no tanque de armazenamento foi realizada com agitador mecânico (não mostrado). As vazões mássicas das correntes de overflow e underflow foram 
determinadas através de medidas de massa cronometradas. A vazão de alimentação foi calculada pelo somatório das vazões mássicas do underflow e overflow. Para análise de sólidos totais, foram coletadas alíquotas em cada uma das correntes (alimentação, overflow e underflow) e suas massas foram medidas antes e após a secagem em estufa a $90^{\circ} \mathrm{C}$ por $2 \mathrm{~h}$.

A eficiência total de separação foi calculada pela razão entre a massa total de sólido coletada no underflow (Wsu) e a massa total de sólido coletada na alimentação (Wsa), conforme Equação 1.

$$
\mathrm{ET}(\%)=\left(\frac{\mathrm{W}_{\mathrm{su}}}{\mathrm{W}_{\mathrm{sa}}}\right) \times 100
$$

A razão de líquido $(\mathrm{RL})$ foi calculada com as informações da vazão volumétrica do fluído que sai na corrente do underflow ( $Q u)$ e a que entra na corrente de alimentação ( $(a)$, conforme a Equação 2. Nessa equação, Cvu é a concentração volumétrica no underflow e, Cva a concentração volumétrica na alimentação.

$$
\mathrm{RL}=\frac{\mathrm{Q}_{\mathrm{u}}\left(1-\mathrm{C}_{\mathrm{vu}}\right)}{\mathrm{Q}_{\mathrm{a}}\left(1-\mathrm{C}_{\mathrm{va}}\right)}
$$

Enquanto que a eficiência total considera todas as partículas coletadas no underflow, a eficiência total reduzida (ET') considera apenas aquelas coletadas no underflow pelo efeito do campo centrífugo. Dessa forma, a influência da divisão de fluxo deve ser desconsiderada e subtraída da eficiência total (ET). Para quantificação desse parâmetro, utilizou-se a Equação 3.

$$
\mathrm{ET}^{\prime}(\%)=\left(\frac{\mathrm{ET}-\mathrm{RL}}{1-\mathrm{RL}}\right) \times 100
$$

\section{RESULTADOS E DISCUSSÃO}

Os resultados de caracterização do mosto de cerveja mostraram que o pH estava na faixa de $5-6$, a densidade em $1,075 \mathrm{~g} / \mathrm{cm}^{3}$ e o teor de sólidos entre 2,9-8,4 \% $\mathrm{m} / \mathrm{m}$. Esses resultados mostram a baixa densidade das leveduras e a heterogeneidade no tamanho dos sólidos no mosto. Na Tabela 2 são apresentados os teores de sólido, a razão de líquido e a eficiência total de separação para os mini-hidrociclones $\mathrm{HC} 090, \mathrm{HC100}, \mathrm{HC1} 10$ e HC120, avaliados em diferentes vazões de alimentação. 
Tabela 2 - Desempenho dos hidrociclones para as diferentes vazões.

\begin{tabular}{|c|c|c|c|c|c|c|c|}
\hline Hidrociclone & $\begin{array}{c}\mathrm{Qa} \\
(\mathrm{Kg} / \mathrm{min})\end{array}$ & $\begin{array}{c}Q u \\
(K g / m i n)\end{array}$ & $\begin{array}{c}P \\
(p s i)\end{array}$ & $\begin{array}{l}\text { Tsa } \\
(\%)\end{array}$ & $\begin{array}{l}\text { Tsu } \\
(\%)\end{array}$ & $\mathbf{R L}$ & $\begin{array}{l}\text { ET } \\
\text { (\%) }\end{array}$ \\
\hline \multirow{5}{*}{ HCO90 } & 1,68 & 1,08 & 30,0 & 4,65 & 3,35 & 0,42 & 72 \\
\hline & 1,16 & 1,04 & 26,5 & 8,15 & 5,13 & 0,52 & 63 \\
\hline & 0,83 & 0,71 & 16,3 & 4,96 & 4,59 & 0,77 & 93 \\
\hline & 0,42 & 0,34 & 4,0 & 3,08 & 2,24 & 0,49 & 73 \\
\hline & 0,21 & 0,20 & 3,3 & 4,89 & 2,45 & 0,13 & 50 \\
\hline \multirow{5}{*}{$\mathrm{HCl} 100$} & 1,57 & 1,18 & 32,0 & 3,16 & 2,99 & 0,14 & 95 \\
\hline & 1,20 & 1,09 & 25,0 & 4,70 & 3,77 & 0,16 & 80 \\
\hline & 0,80 & 0,52 & 8,0 & 3,40 & 2,33 & 0,41 & 79 \\
\hline & 0,40 & 0,21 & 2,4 & 4,18 & 3,29 & 0,50 & 72 \\
\hline & 0,19 & 0,15 & 1,7 & 2,92 & 1,03 & 0,01 & 35 \\
\hline \multirow{5}{*}{$\mathrm{HC} 110$} & 1,70 & 1,59 & 24,0 & 5,50 & 3,79 & 0,56 & 76 \\
\hline & 1,22 & 1,11 & 18,0 & 5,78 & 3,94 & 0,56 & 79 \\
\hline & 0,77 & 0,60 & 8,0 & 5,40 & 4,10 & 0,53 & 57 \\
\hline & 0,48 & 0,35 & 3,5 & 5,60 & 3,99 & 0,47 & 51 \\
\hline & 0,25 & 0,15 & 1,5 & 4,05 & 3,11 & 0,39 & 43 \\
\hline \multirow{5}{*}{$\mathrm{HC} 120$} & 1,67 & 0,86 & 22,0 & 5,75 & 3,24 & 0,16 & 56 \\
\hline & 1,28 & 0,69 & 16,0 & 3,23 & 3,10 & 0,09 & 96 \\
\hline & 0,82 & 0,51 & 8,0 & 2,59 & 2,25 & 0,07 & 87 \\
\hline & 0,45 & 0,29 & 3,5 & 2,90 & 1,03 & 0,03 & 36 \\
\hline & 0,19 & 0,10 & 1,1 & 3,28 & 1,08 & 0,04 & 33 \\
\hline
\end{tabular}

$\mathrm{Q} a$ = vazão mássica na alimentação, $\mathrm{Qu}=$ vazão mássica no underflow, $\mathrm{P}=$ Pressão de alimentação, Tsa = teor de sólidos na alimentação, Tsu = teor de sólidos no underflow, RL = razão de líquido, ET= eficiência total.

Fonte: dos autores, 2018.

Os dados da Tabela 2 mostram que o aumento da vazão de alimentação nos mini-hidrociclones levou ao aumento da vazão no underflow e, consequentemente, ao aumento da pressão. Pode-se observar que os dados de eficiência total de separação variam amplamente para os hidrociclones HC090 (50-93\%), HC100 (35-95\%), HC110 (43-63\%) e HC120 (33-96\%). Essas variações na eficiência total podem ser atribuídas à formação de espuma e cavitação da bomba que também foram observadas por outros pesquisadores (BICALHO, 2011; MOGNON, 2015).

A Figura 3 apresenta os resultados da eficiência total reduzida (ET') de separação de sólidos em função da vazão de alimentação nos hidrociclones HC90, HC100, HC110 e HC120. Nota-se que, nas condições experimentais utilizadas, o hidrociclone $\mathrm{HC} 120$ apresentou as menores eficiências reduzidas $(<8 \%)$, que podem ser atribuídas à turbulência nos fluxos do underflow e imperfeições no vórtex no interior do equipamento, resultando em partículas maiores saindo pelo overflow. Esse efeito ocorre devido às maiores dimensões desse hidrociclone $(\mathrm{HC120})$ e, portanto, seriam necessárias vazões maiores para a formação do vórtex interno, responsável pelo mecanismo de separação em hidrociclones (NI et al., 2018). 
Figura 3 - Eficiência total reduzida em função da vazão mássica de alimentação para os hidrociclones $\mathrm{HC} 090, \mathrm{HC} 100, \mathrm{HC} 110$ e $\mathrm{HC} 120$.

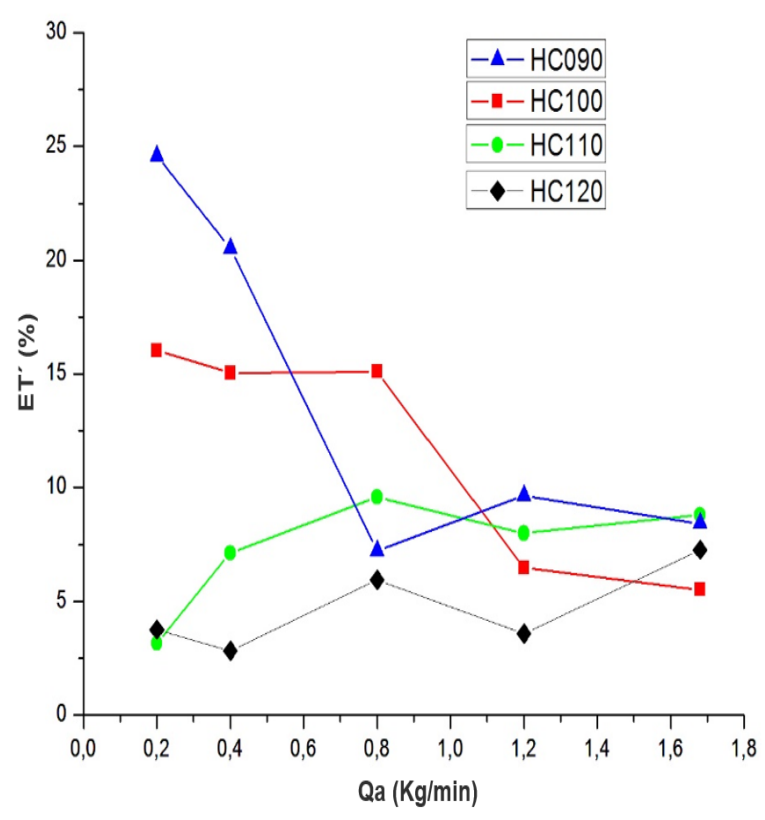

Fonte: dos autores, 2018.

A Figura 3 mostra também que os hidrociclones HC090 e HC100 apresentam as maiores eficiências totais reduzidas para separação de leveduras. Para maximizar a eficiência de separação, devem ser utilizados hidrociclones com baixa relação Do/Dc e alta relação Du/Dc (BICALHO, 2012; PINTO et al., 2008; SALVO et al., 2013). As relações Do/Dc e Du/Dc dos mini-hidrociclones são apresentados na Tabela 3. Nota-se que a relação Do/Dc dos hidrociclones pode ser considerada igual, mas a relação $\mathrm{Du} / \mathrm{Dc}$ apresenta a seguinte ordem: $\mathrm{HC} 090>\mathrm{HC} 100>\mathrm{HC} 110>\mathrm{HC1} 20$. Constata-se também que quanto maior a relação Du/Dc maior a eficiência reduzida máxima. Logo, os hidrociclones HC090 e HC100 apresentaram o melhor desempenho para baixas vazões mássicas.

Tabela 3 - Características e eficiência dos mini-hidrociclones.

\begin{tabular}{cccc}
\hline Hidrociclone & Do/Dc & Du/Dc & ET`máxima \\
\hline HC090 & 0,21 & 0,18 & 24,59 \\
HC100 & 0,21 & 0,16 & 16,19 \\
HC110 & 0,22 & 0,14 & 9,72 \\
HC120 & 0,22 & 0,13 & 7,27 \\
\hline
\end{tabular}

${ }^{\mathrm{a} E T}$ 'maxima = eficiência total reduzida máxima.

Fonte: dos autores, 2018 


\section{CONCLUSÕES}

Os resultados evidenciaram que a impressão 3D de mini-hidrociclones da família Mozley1, em plástico $A B S$, é uma técnica rápida e barata para se construir peças e equipamentos de estruturas complexas para experimentos de engenharia. Foi observado que o aumento da vazão de alimentação levou ao aumento da vazão no underflow e da pressão nos hidrociclones. A eficiência total de separação variou amplamente para os hidrociclones $\mathrm{HCO} 00$ (50-93\%), HC100 (35-95\%), HC1 10 (43-79\%) e HC120 (33-96\%). A eficiência total reduzida está relacionada com a relação Du/Dc de construção dos hidrociclones, ou seja, quanto maior a relação Du/Dc maior a eficiência reduzida máxima. Os hidrociclones, que se mostram promissores para a separação de leveduras do mosto da indústria cervejeira, apresentaram a seguinte ordem de eficiência na separação de leveduras de cerveja: $\mathrm{HCO} 0>\mathrm{HC} 100>\mathrm{HC1} 10>\mathrm{HC} 120$.

\section{AGRADECIMENTOS}

As Cervejarias Walls e Loba, pelo fornecimento do mosto de cerveja. 


\section{REFERÊNCIAS}

ARAÚJO, C. A. O.; SCHEID, C. M.; KLEIN, T. S.; LOUREIRO, J. B. R.; MEDRONHO, R.A. Eficiência de separação de hidrociclones para a separação de mistura óleo-água com alto teor de óleo: Comparação entre resultados simulados e experimentais. Anais do XXXVII Congresso Brasileiro de Sistemas Particulados - ENEMP, São Paulo, Brasil, p. 958-965, 2015.

BICALHO, Isabele Cristina. Concentração de leveduras da fermentação alcoólica em hidrociclones. Dissertação (M. Sc.), Universidade Federal de Uberlândia, Uberlândia, MG, Brasil, 2011.

BICALHO, I. C.; MOGNON, J. L.; SHIMOYAMA, J.; ATAÍDE, C. H.; DUARTE, C. R. Separation of yeast from alcoholic fermentation in small hydrocyclones. Separation and Purification Technology, v. 87, p. 62-70, 2012.

CERVBRASIL - Associação Brasileira da Indústria da Cerveja. Dados do setor cervejeiro. Disponível em: $<$ http://www.cervbrasil.org.br/paginas/index.php?page=dados-do-setor $>$. Acessado em maio de 2016.

EVANS, W. K.; ONKAEW, P.; HEUNGHOK, B. An Application of small hydrocyclones for separating yeast in the brewing industry. Asian Journal of Engineering and Technology, v.3, p.2321-2462, 2015.

MOGNON, José Lucas. Mini-hidrociclones aplicados ao sistema de controle de sólidos na perfuração de poços de petróleo. Dissertação (M. Sc.), Universidade Federal de Uberlândia, Uberlândia, MG, Brasil, 2015.

$\mathrm{NI}, \mathrm{L} . ;$ TIAN, J.; ZHAO, J. Experimental study of the relationship between separation performance and lengths of vortex finder of a novel de-foulant hydrocyclone with continuous underflow and reflux function. Separation Science and Technology, 52(1), p.142-154, 2017.

$\mathrm{NI}$, L.; TIAN, J.; SONG, T.; JONG, Y.; ZHAO, J. Optimizing geometric parameters in hydrocyclones for enhanced separations: a review and perspective. Separation \& Purification Reviews, in press, p. 1-22, 2018.

PINTO, R. C. V.; MEDRONHO R. A.; CASTILHO L. R. Separation of $\mathrm{CHO}$ cells using hydrocyclones. Cytotechnology, 56, p. 57-67, 2008.

SALVO, R. V.; SOUZA, F. J.; MARTINS, D. A. M. Effects of outlet duct length and shape on the performance of cyclone separators. $22^{\text {nd }}$ Internacional Congress of Mechanical Engineering - COBEM, Ribeirão Preto, São Paulo, Brasil, p. 1688-1699, 2013.

SYED, M.; RAFEIE, M.; HENDERSON, R.; ASADNIA, M.; WARKIANI, M.E. A 3D-printed mini-hydrocyclone for high throughput particle separation: application to primary harvesting of microalgae. Lab Chip, $v$. 17, p. 2459-2469, 2017.

YU, J.F.; FU, J.; CHENG, H.; CUI, Z. Recycling of rare earth particle by mini-hydrocyclones. Waste Management, v. 61, p. 362-371, 2017. 